\title{
Systematic construction of global socioeconomic pathways using internally consistent element combinations
}

\author{
Vanessa J. Schweizer • Brian C. O’Neill
}

Received: 21 January 2013 / Accepted: 27 August 2013 / Published online: 2 October 2013

(C) The Author(s) 2013. This article is published with open access at Springerlink.com

\begin{abstract}
Shared Socioeconomic Pathways (SSPs) describe alternative outcomes for socioeconomic development. Papers describing the conceptual framework for SSPs refer to challenges to mitigation and to adaptation as fundamental concepts. Identifying which socioeconomic factors are the most important determinants of these challenges, and how to combine them in an internally consistent manner, is critical to scenario design. Here we demonstrate a systematic and traceable approach for identifying and prioritizing scenario elements. In this study, we identify 13 determinants of mitigation and adaptation challenges at a globally aggregated scale based on a survey of 25 experts. In addition, we use 19 expert elicitations and a cross-impact balance analysis to create approximately 1.5 million combinations of trends for these determinants and rank them in terms of internal consistency. Using the 1,000 most consistent combinations, we construct composite metrics for challenges to mitigation and adaptation to uncover distinguishable characteristics for five types of SSPs: those with Low, Medium, and High challenges to both mitigation and adaptation (consistent with SSPs 1-3), and those in which adaptation challenges or mitigation challenges dominate (consistent with SSPs 4-5). We find a distinguishing characteristic for mixed typology SSP4 (low mitigation challenges, high adaptation challenges): High trends for innovation capacity could lower challenges to mitigation but not necessarily challenges to adaptation. We also find that a low trend for quality of governance consistently corresponds to higher challenges to adaptation. These findings are suggestive for future research on the SSPs in particular, while our analytical approach is instructive for scenario development in general.
\end{abstract}

This article is part of the Special Issue on "A Framework for the Development of New Socioeconomic Scenarios for Climate Change Research" edited by Nebojsa Nakicenovic, Robert Lempert, and Anthony Janetos.

Electronic supplementary material The online version of this article (doi:10.1007/s10584-013-0908-z) contains supplementary material, which is available to authorized users.

V. J. Schweizer $(\bowtie) \cdot$ B. C. O’Neill

National Center for Atmospheric Research, P.O. Box 3000, Boulder, CO 80307, USA

e-mail: vjs@alumni.cmu.edu 


\section{Introduction}

A process is underway to develop new scenarios for future climate change research (Ebi et al. 2013; Moss et al. 2010). This process involves the efforts of different climate research communities working in parallel. Climate modeling comprises the first track, while research communities who study integrated assessment modeling (IAM) as well as impacts, adaptation, and vulnerability (IAV) comprise the second track. Ultimately, the two tracks will be brought together for complete, integrated analyses of climate change impacts and policy responses. Here we focus on the second track, where the community activity is aimed at developing a small number of alternative possible trends in future socioeconomic development, referred to as shared socioeconomic pathways (SSPs; O'Neill et al. 2013). SSPs could be paired with different trajectories of radiative forcing known as representative concentration pathways (RCPs; van Vuuren et al. 2013, 2011) and with common policy assumptions (Kriegler et al., Submitted for publication in this special issue).

In environmental change assessments, scenarios depicting socioeconomic developments often include qualitative and quantitative factors, which are referred to as scenario elements (Alcamo 2008; Rounsevell and Metzger 2010). The SSPs will be no exception. Because of the many uncertainties involved in socioeconomic change over many decades, qualitative elements are necessary to represent factors that could influence long-term developments but may be difficult to represent formally in quantitative models. The conceptual framework for the SSPs suggests that the logic of the socioeconomic components of new scenarios should be rooted in the concepts of socioeconomic challenges to mitigation and challenges to adaptation (O'Neill et al. 2013). SSPs would describe baseline, or reference, cases for the future, i.e., alternative socioeconomic developments that could increase or decrease challenges to mitigation or adaptation regardless of climate change outcomes or climate policy. Additionally, there will be five types of SSPs, which correspond to different combinations of Low, Medium, or High challenges to mitigation and adaptation (O'Neill et al. 2012).

A number of key questions remain for fleshing out SSPs, and we address four in this paper. First, what socioeconomic factors would be most important to include as elements in new scenarios to distinguish challenges to mitigation and to adaptation? The conceptual framework suggests that qualitative elements for challenges to mitigation should incorporate (1) socioeconomic developments that tend to drive lower or higher baseline emissions, such as demographic trends and the carbon intensity of energy portfolios, and (2) socioeconomic developments that tend to determine lower or higher mitigative capacity, such as the rate of technological change. Similarly, qualitative elements for challenges to adaptation should incorporate (1) socioeconomic developments that tend to characterize lower or higher socioeconomic exposure and sensitivity (Smit and Wandel 2006), such as concentrations of population in vulnerable areas, e.g. coasts, and (2) socioeconomic developments that tend to characterize lower or higher adaptive capacity, such as quality of governance or level of economic development. However, no decisive list of socioeconomic elements has been identified for either axis.

Second, even after specific socioeconomic elements have been identified, how could different trends across these elements be combined to derive new qualitative scenarios that are internally consistent? Traditionally, IAM would serve as the primary way to verify or test the consistency of qualitative socioeconomic scenarios via simulation (Alcamo and Henrichs 2008; O'Neill et al. 2013). However, IAM does not always include all elements that appear in qualitative scenario descriptions. Furthermore, depending upon the richness of new socioeconomic scenarios, there could be a very large number of possible combinations of socioeconomic elements. It may be unrealistic to expect IAM to assess the internal 
consistency of the full range of scenario possibilities comprehensively. Are there other analytical tools that could augment assessments of internal consistency?

Third, once internally consistent combinations of socioeconomic trends have been derived, how could any particular combination be classified as consistent with one of the five types of the SSPs? Socioeconomic pathways will contain information about long-term trends for a variety of elements. Are any particular trends (or some combination) indicative of pathways with higher or lower challenges to mitigation or adaptation? Answering these questions will require the development and application of a set of metrics for challenges to mitigation and to adaptation in order to classify specific trend combinations.

Fourth, could the classification of multiple socioeconomic pathways enable a metaanalysis that reveals common, distinguishable characteristics that could be used to construct SSP archetypes? Studying samples of specific socioeconomic pathways and identifying their shared characteristics could helpfully document representative assumptions for alternative futures. Such meta-analyses may guard against political challenges to the SSPs of the kind encountered by the scenario typologies of the Special Report on Emissions Scenarios (SRES; Nakicenovic et al. 2000) published by the Intergovernmental Panel on Climate Change (IPCC; see discussion of political challenges in Girod and Flueeler 2009).

In this paper, we address the above questions and demonstrate a systematic and traceable approach for developing new socioeconomic pathways at the globally aggregated scale. Each section of the paper treats one of the four key questions described above. In section 2, we describe our methods and findings for an expert survey to address the first question, which identified prioritized lists of socioeconomic elements relevant for challenges to mitigation and adaptation. We then used these findings to address the second question, the derivation of internally consistent qualitative scenarios (socioeconomic pathways), which is summarized in section 3. In section 4, we report how we addressed the third question, socioeconomic pathway classification, by developing metrics for challenges to mitigation and to adaptation so that cases of socioeconomic pathways could be binned according to five domains that can be constructed for a "challenges space." In section 5, we investigated pathways within each domain to uncover representative, distinguishable characteristics for each archetype, which were then used to sketch possible SSPs. Finally, in section 6, we discuss conclusions from our findings across the four key questions that are suggestive for the continued development of the SSPs. We also comment on how this analytical approach can be instructive for scenario research in general.

\section{Socioeconomic elements for challenges to mitigation and to adaptation}

Before scenarios can be developed, decisions must be made about which socioeconomic factors to prioritize as scenario elements. We addressed this need with an Internet-based survey. This survey was sent to 74 experts 8 weeks before the Boulder 2011 Workshop on The Nature and Use of New Socioeconomic Pathways for Climate Change Research (O'Neill et al. 2012) with the intention that preliminary results might inform discussion of prototype SSPs. Experts were invited who have contributed to the new scenario process, do research in IAM, or do research in IAV. Names of experts were obtained from attendee lists to scientific meetings regarding new socioeconomic scenarios or lists of lead authors for the IPCC Fifth Assessment Report. We focused on lead authors for Working Groups II and III.

The survey asked experts to select socioeconomic factors they believed were most important for determining challenges to adaptation or mitigation at a globally aggregated scale. Factors prioritized by the most experts became scenario elements for our study. 
Further discussions regarding the design and implementation of the survey are in Online Resource 1, and a copy of the survey instrument is in Online Resource 2.

Responses received no later than 4 weeks prior to the Boulder workshop were used to identify socioeconomic elements that we would use in our construction of internally consistent socioeconomic pathways (detailed below in section 3). Twenty-five (25) experts responded in time (names of expert respondents are in Online Resource 1), so the response rate should be considered $34 \%$. Socioeconomic elements that these experts prioritized are summarized below in bulleted lists. In this section, the categorization of elements as relevant for challenges to mitigation or adaptation reflects their presentation in the survey, e.g. income per capita was presented to experts answering questions about either socioeconomic challenges to mitigation or adaptation. Percentages next to each element indicate the percentage of respondents who prioritized it. They do not add up to $100 \%$, as experts were able to prioritize multiple elements. Socioeconomic factors not shown in the lists were selected by fewer than $25 \%$ of respondents and were not included in scenarios for the remainder of our study.

Socioeconomic factors relevant for challenges to mitigation and for challenges to adaptation

- $\quad$ Income per capita (84\%)

Socioeconomic factors relevant for challenges to mitigation

- Population (68\%)

- Carbon intensity (44\%)

- Energy-related technological change (44\%)

- Agricultural productivity (40\%)

- Energy intensity (36\%)

Socioeconomic factors relevant for challenges to adaptation

- Extreme poverty (76\%)

- Quality of governance (52\%)

- Water scarcity (46\%)

- Proportion of population on coasts (44\%)

- Innovation capacity (36\%)

- Urbanization (32\%)

- Educational attainment $(24 \%)^{1}$

\section{Internally consistent combinations of socioeconomic trends}

The prioritized socioeconomic elements from the expert survey served as inputs for the derivation of internally consistent socioeconomic pathways. We used a systematic technique, cross-impact balance (CIB) analysis (Weimer-Jehle 2006), to generate a large number of scenarios comprising combinations of possible trends for these elements. This method requires judgments for how scenario elements interrelate, which we collected from additional experts during face-to-face workshops or through a combination of email and telephone contact. To understand our decisions for how we constructed our expert elicitation instrument, we provide a brief overview of CIB analysis here. Detailed descriptions can be

\footnotetext{
${ }^{1}$ Despite the small percentage of respondents who chose this element, we opted to include it, as there was substantial discussion about its inclusion in SSPs during a 2011 workshop in Changwon City. See IAMC (2012).
} 
found in Weimer-Jehle (2006) and Schweizer and Kriegler (2012), which applied CIB to the SRES. Further detail on CIB is also in Online Resource 1.

\subsection{Overview of the CIB method}

As discussed in section 1, scenarios contain quantitative and qualitative information about the developments of factors over time. Such information will henceforth be referred to as outcomes for elements of a scenario. CIB analysis is a systematic method for identifying internally consistent combinations of outcomes for elements that qualitatively describe scenarios. In CIB analysis, all possible combinations are scored for internal consistency. Consistency scoring is determined by a collection of expert judgments regarding how each outcome specified for some element would be expected to directly influence the outcomes of other elements. In CIB analysis, each scenario element is associated with a set of direct influences on other elements. A combination of outcomes that evokes a set of direct influences reinforcing the original combination (rather than supporting an alternative combination) is said to be self-consistent and is therefore deemed internally consistent. Thus in CIB analysis, internal consistency for any combination of element outcomes is determined by a logical check for self-consistency (Weimer-Jehle 2006). Self-consistency is an important property for stable scenarios (von Reibnitz 1988), or scenarios that describe long-term trends, which is precisely what the SSPs aim to do.

A simple example of self-consistency can be constructed for a scenario consisting of three elements: population, income per capita, and educational attainment (see Online Resource 1 for more detailed discussion of this example). A scenario defined by the combination of a high outcome for educational attainment, high income per capita, and low population would be selfconsistent because the direct influences among these outcomes are relatively well understood and reinforce the combination. Such a self-consistent scenario describes a set of long-term trends. As shown in Fig. 1a, when global educational attainment outcomes are high, one could expect the outcome for average global income per capita to also be high. When the outcomes for both income and educational attainment are high, one could expect the global population outcome to be low. Low outcomes for global population might also weakly encourage a high outcome for income per capita on a global average. The high outcome for income per capita also encourages continued high educational attainment. Thus the outcome for each element reinforces the outcomes for the others, and the scenario is self-consistent. In contrast, if one considers the same scenario but with the outcome for educational attainment changed to low (Fig. 1b), the scenario is no longer self-consistent. A low global outcome for educational attainment would not be expected to encourage the high outcome for average global income nor the low outcome for global population. Similarly, a high outcome for global income per capita would not be expected to encourage a low outcome for educational attainment.

In addition to identifying internally consistent and inconsistent scenarios, CIB analysis also scores the degree of internal inconsistency for a scenario. Internally inconsistent scenarios have one or more relationships between elements that are not self-consistent. CIB analysis assigns an inconsistency score by measuring the worst discrepancy between element relationships specified in the scenario and self-consistent element relationships. Thus perfectly internally consistent scenarios have an inconsistency score of 0 . Measurement of internal consistency is made possible by introducing quantities that represent the expected direction of influences (i.e. a given trend for socioeconomic element $x_{i}$ may encourage or discourage a target outcome for socioeconomic element $y_{i}$ ) as well as magnitude (i.e. a given trend for $x_{i}$ may exert a very strong or weak influence on the target outcome for $y_{i}$ ). For further details of such quantification, see Online Resource 1. 

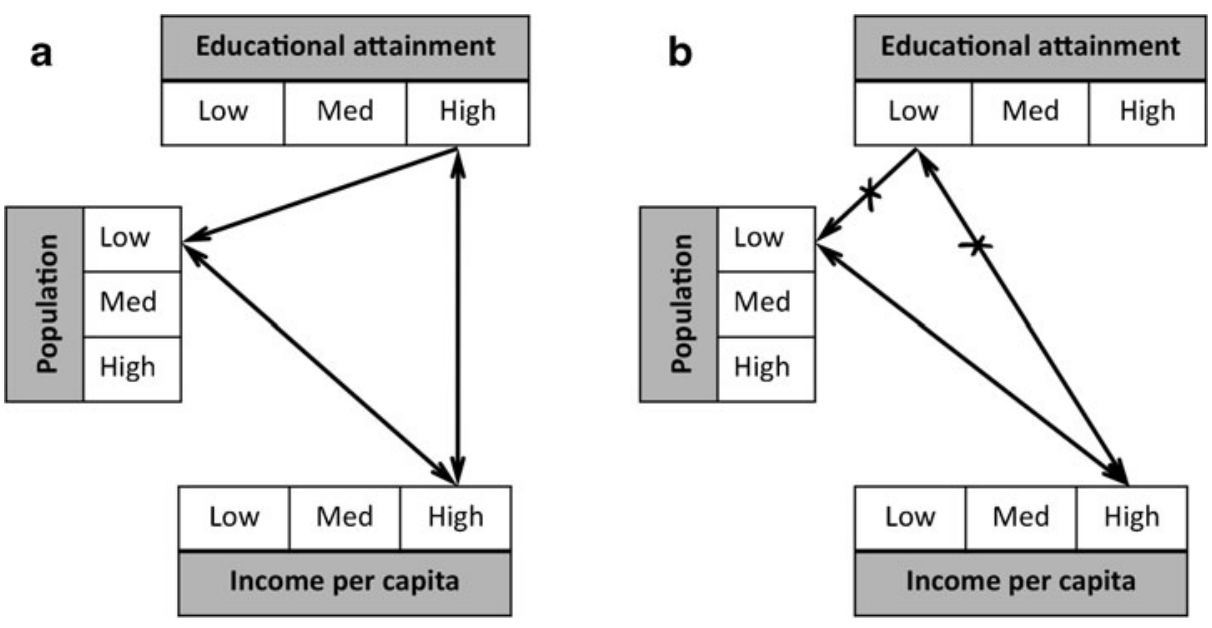

Fig. 1 Two cases of possible combinations of outcomes for the socioeconomic elements educational attainment, population, and income per capita conceptualized at the global scale. The combination at left (a) is an example of an internally consistent (self-consistent) case. The combination at right (b) is internally inconsistent, as the outcome combinations are not self-consistent

\subsection{Elicitation instrument and protocol}

Elicitations of 19 experts were performed to obtain judgments about relationships between outcomes for the 13 prioritized socioeconomic elements (names of experts elicited and a description of how they were recruited are in Online Resource 1). We prepared a standard set of materials that would define each socioeconomic element as well as the outcomes of low, medium, or high trends (pathways). These materials were supported by scholarly literature and contained definitions, quantitative ranges, and, where possible, quantitative projections. In some cases, projections for trends over the 21 st century were developed from empirical data available from the United Nations, the World Bank, the International Energy Agency, the US Department of Agriculture, the International Institute for Applied Systems Analysis, and the World Resources Institute. Across the 13 elements, 10 could be represented quantitatively, while three (rate of energy-related technological change, quality of governance, and innovation capacity) were represented qualitatively (Online Resources 3 and 4 contain further information).

The number of judgments required for this study is large $(13 \times 13$ socioeconomic elements, each with three possible outcomes; this requires 1,404 judgments), and it draws upon scholarship across many disciplines. For this reason, we consulted experts in different fields and asked them to provide judgments relevant to their expertise, which covered only a fraction (108-216 judgments) of the total judgments needed. Nevertheless, these judgments, which were collected from experts independently, could be synthesized in the $\mathrm{CIB}$ analysis.

Expert judgments were rooted in the following two-part cross-impact question (WeimerJehle 2006), which was applied to each outcome for each given socioeconomic element $x_{i}$ that could directly influence some target socioeconomic element $y_{i}$.

(a) Knowing nothing else about the outcomes of other socioeconomic elements, and in comparison to having no information about the outcome of socioeconomic element $x_{i}$, 
would you expect the given pathway for $x_{i}$ to make the target outcome for socioeconomic element $y_{i}$ more likely, less likely, or neither?

(b) If the given pathway would be expected to affect the target outcome, how important is the direct influence of the given pathway for $x_{i}$ on the target outcome for $y_{i}$ ? Does it affect the target outcome very much, moderately, or slightly?

Experts were briefed to report their judgments according to a discrete seven-point scale (e.g. judgment scores ranging from +3 to -3 ). ${ }^{2}$ Further detail on guidance provided to experts for their judgments can be found in Online Resource 3. All experts in the workshop setting were able to provide 108 judgments for the CIB matrix in an hour or less. ${ }^{3}$

\subsection{Internal consistency scoring of all possible outcome combinations}

For socioeconomic scenarios consisting of 13 elements, with each element having three possible outcomes, there are over 1.5 million possible ways to combine outcomes $\left(3^{13}=1,594,323\right)$. Using the CIB software package ScenarioWizard, we calculated inconsistency scores for each combination and constructed a frequency distribution (see figure ESM1-2 in Online Resource 1). For many socioeconomic elements, we collected judgments from more than one expert. It was thus possible to split the judgments into two expert panels (A and B) to better explore the sensitivity of results to differences in expert opinion as discussed in sections 5 and 6 . For both panels, combinations with the most severe internal inconsistencies had inconsistency scores $\geq 93$. Inconsistency scores are ordinal, and the threshold for acceptable levels of internal inconsistency are determined by the scenario analyst. Because there were so many possible combinations for the outcomes of the socioeconomic elements, for this study, we were less interested in setting an internal consistency threshold. Instead, we isolated the 1,000 combinations with the best scores for internal consistency to uncover any distinctive patterns among these combinations. For both panels, the first 1,000 combinations that performed the best had internal inconsistency scores ranging from 0 to 9 .

\section{Metrics for classifying scenarios into five SSP typologies}

Challenges to mitigation or to adaptation can be considered as two dimensions of a "challenges" space (O’Neill et al. 2013) consisting of five domains: co-varying challenges to mitigation and adaptation that are (1) Low, (2) Medium, or (3) High, and anti-correlated challenges where (4) Adaptation challenges dominate or (5) Mitigation challenges dominate. Using the 1,000 internally consistent combinations identified by the CIB analysis, we classified or binned each combination into one of the five domains. We developed composite metrics for each challenges dimension (mitigation versus adaptation) so that coordinates in the challenges space could be assigned to each combination of element outcomes.

\footnotetext{
${ }^{2}$ Part (a) of the cross-impact question corresponds to the sign of the judgment in the cross-impact matrix $(+,-$, 0 ). Part (b) of the question corresponds to the magnitude of the judgment $(3,2,1)$.

${ }^{3}$ Because the remote elicitation was asynchronous, the time it took experts to complete the questionnaire cannot be determined. However, we assume the cognitive burdens for remote elicitation were comparable to the workshop setting.
} 


\subsection{Developing metrics}

Metrics for challenges to mitigation and challenges to adaptation were developed through an iterative process, which is elaborated in Online Resource 5. Iteration was necessary because we found that different assumptions for metrics could alter the classification of scenarios. It should be noted that through these iterations, we arrived at different lists of elements that determine challenges to mitigation and adaptation compared to what was presented in the expert survey discussed in section 2. An abridged description of our process is that, first, we (the authors) evaluated how much of a challenge each combination represented for mitigation or adaptation. This was done by assigning a socioeconomic element to challenges to mitigation, challenges to adaptation, or to both. Then each outcome for each element was interpreted as posing a low, medium, or high challenge, and an ordinal value of 1-3 (1 for low challenges, 3 for high challenges) was assigned to the outcome. Table 1 summarizes our final decisions for how outcomes for each socioeconomic element were evaluated for the challenge axes.

For developing composite metrics for each axis, we began with interpreting each socioeconomic element as equally important for challenges to mitigation or to adaptation. Mathematical representations for the challenge metrics are as follows.

Metric for challenges to adaptation:

$$
A_{1}=L+Z+V+W+H+G+I
$$

where

$A_{1} \quad$ Adaptation challenges metric, based on elements weighted equally

$L \quad$ Income per capita challenge value

$Z \quad$ Agricultural productivity challenge value

$V \quad$ Extreme poverty challenge value

$W \quad$ Water scarcity challenge value

$H \quad$ Educational attainment challenge value

$G \quad$ Governance quality challenge value

I Innovation capacity challenge value

Table 1 Evaluation of element outcomes for challenges to mitigation or adaptation. Outcomes for agricultural productivity were interpreted as relevant for challenges to mitigation and to adaptation as detailed in Online Resource 5

\begin{tabular}{|c|c|c|}
\hline Challenges: & Adaptation (horizontal axis) & Mitigation (vertical axis) \\
\hline Low (value=1) & $\begin{array}{l}\text { Low outcomes: Extreme poverty, water scarcity } \\
\text { High outcomes: Income, educational attainment, } \\
\text { governance, innovation, agricultural productivity }\end{array}$ & $\begin{array}{l}\text { Low outcomes: Population, energy } \\
\text { intensity, carbon intensity } \\
\text { High outcomes: Technological } \\
\text { change, agricultural productivity }\end{array}$ \\
\hline Medium $($ value $=2$ ) & Any medium outcomes & Any medium outcomes \\
\hline High $($ value $=3$ ) & $\begin{array}{l}\text { Low outcomes: Income, educational attainment, } \\
\text { governance, innovation, agricultural productivity } \\
\text { High outcomes: Extreme poverty, water scarcity }\end{array}$ & $\begin{array}{l}\text { Low outcomes: Technological } \\
\text { change, agricultural productivity } \\
\text { High outcomes: Population, energy } \\
\text { intensity, carbon intensity }\end{array}$ \\
\hline
\end{tabular}


Metric for challenges to mitigation:

$$
M_{1}=P+E+C+T+Z
$$

where

$M_{1} \quad$ Mitigation challenges metric, based on elements weighted equally

$P \quad$ Population challenge value

$E \quad$ Energy intensity challenge value

$C$ Carbon intensity challenge value

$T$ Technological change challenge value

$Z \quad$ Agricultural productivity challenge value

We also considered metrics where socioeconomic elements were weighted differently. In effect, differential weights represent the case in which some socioeconomic elements are considered more important than others for mitigation or adaptation challenges. ${ }^{4}$

After these metrics were obtained for each axis for all 1,000 combinations, the metrics were normalized to arrive at coordinates for each axis between 0 and 1 . Thus the axes for the challenges space are normalized such that the lowest challenges have coordinates $(0.33$, $0.33)$, while maximum challenge coordinates are $(1.0,1.0) .^{5}$

\subsection{Boundaries for bins in the challenges space}

Since combinations had domain and range $[0.33,1.0]$, boundaries for the bins were drawn around the 1,000 combinations. The five bins were interpreted as non-overlapping, with the central bin, Medium challenges, corresponding to the middle third of the interval $[0.33,1.0]$. This interval can be divided into three approximately equal steps at the points $p=0.55$ and $q=0.78$. The corner domains for Low challenges, High challenges, Adaptation challenges dominate, and Mitigation challenges dominate were divided from each other by the midpoint of the interval, $m=0.67$. A formal representation of the five bins can be found in Online Resource 5. A visual representation of the boundaries appears in Fig. 2.

\section{Meta-analysis for distinguishable characteristics of each challenges domain}

Results featured in this paper assume equal weighting for socioeconomic elements for the binning metrics. Additionally, as mentioned previously, we had enough expert judgments to analyze results according to two panels. Results for Panel A are discussed here. Results for Panel B are in Online Resource 5.

The first notable finding is that internally consistent combinations populate all five domains of the challenges space. Most of the combinations (850) lie along the diagonal for Low, Medium, or High challenges to mitigation and adaptation, and most of these lie in Medium and High challenges domains (see Fig. 2; this was also found for Panel B).

\footnotetext{
${ }^{4}$ As discussed in Online Resource 5, we considered a case where extreme poverty, educational attainment, and governance were considered most important for challenges to adaptation, followed by other elements. For challenges to mitigation, we considered a case where energy intensity and carbon intensity were considered most important, followed by other elements.

${ }^{5}$ Although challenge values (Table 1) are ordinal and only one significant figure, most combinations had total challenge scores along each axis that ranged in the tens. Thus two significant figures were retained for indexing.
} 


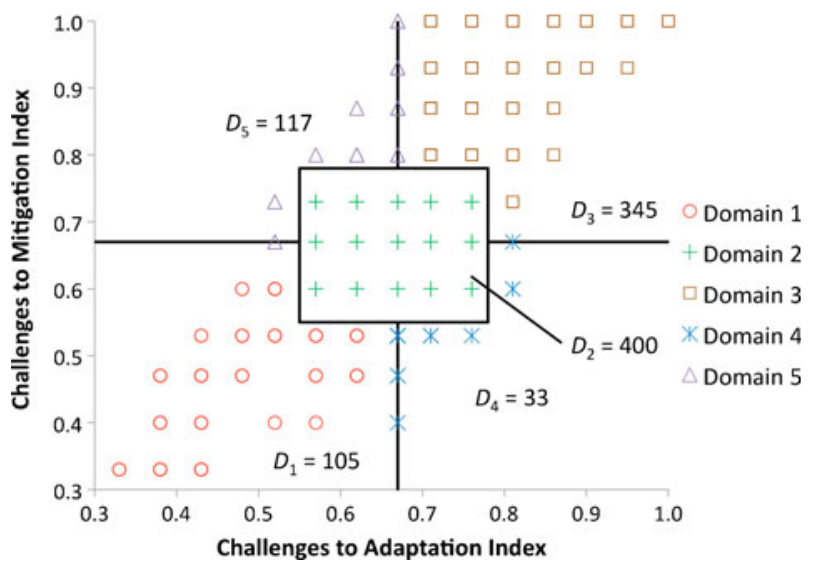

Fig. 2 Map of 1,000 internally consistent element combinations in the challenges space with metrics assuming equal weighting as described in section 4.1. Markers show the coordinates of the 1,000 combinations. Boundaries for the five domains were drawn according to the definition in section 4.2. Counts refer to the number of combinations classified to each domain

Within each domain, there are distinguishable characteristics across the combinations, which are summarized in Fig. 3. In this figure, each row describes a domain type, while each column represents a scenario element. Light cells indicate that the dominant outcome for the scenario element corresponds to a low challenge for mitigation or adaptation, while dark cells correspond to high challenges. Gray cells indicate medium challenges, while a spectrum indicates multiple outcomes (and thus multiple levels of challenges) were internally consistent. Speckled cells indicate elements that were not incorporated into the metrics for measuring challenges. ${ }^{6}$ Arrows summarize the specific outcome for the socioeconomic element. For example, in the cell for challenges domain 1 (row) and the socioeconomic element of population (column), the downward sloping arrow indicates that the low trend was the dominant outcome, which corresponds to a low mitigation challenge (white cell). The cell for challenges domain 2 and the population column has three arrows; this indicates that no outcome was clearly dominant, which also means that the level of mitigation challenge for population for this domain spanned the spectrum of low, medium, and high challenges (spectrum of white, gray, black).

As expected, the challenges domain 1 has the most white cells, challenges domain 2 has the most gray cells, and challenges domain 3 has the most dark cells. The mixed domains (4 and 5) have combinations of white and dark cells. Domains with low challenges to mitigation (1 and 4) exhibit low global trends for population, energy intensity, and carbon intensity. In contrast, domains with high challenges to mitigation (3 and 5) exhibit high global trends for these elements. Medium trend outcomes consistently appeared across these

\footnotetext{
${ }^{6}$ Urbanization and coastal population were not incorporated into metrics because they either introduced noise or would inflate the challenges score for domain classification. Urbanization was considered noisy, since it could either increase challenges to adaptation by concentrating population and assets in potentially vulnerable locations, or it could decrease challenges by potentially enhancing social organization for economic efficiency, emergency response, etc. Coastal population was deemed inflating, as it did not interact much with other elements. This had the effect of providing little reason for the prevalence of low, medium, or high outcomes for coastal population in any particular domain. Including it in the adaptation challenge metric decreased the membership of combinations in domains 1 and 5 and increased the membership of combinations in domain 3 . In our view, these effects were artifacts. For these reasons, we did not incorporate coastal population into metrics for this analysis.
} 


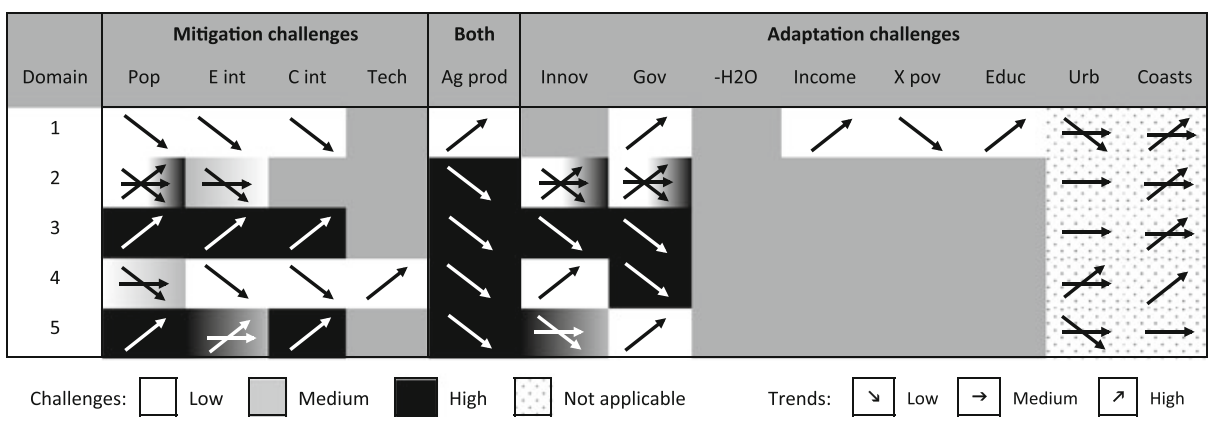

Fig. 3 Summary of distinguishable characteristics for combinations by domain type with metrics assuming equal element weighting. Domain classifications are according to the map shown in Fig. 2. Dominant trends are shown with arrows. Medium challenges cells (gray) correspond to medium trends as dominant (level arrows). For ease of reading, medium trends in the gray cells are not shown. Multiple arrows indicate that more than one dominant trend occurred in the underlying element combinations

elements for challenges domain 2, along with mixed trend outcomes. These findings were generally reproduced for Panel B.

Domains with low challenges to adaptation (1 and 5) exhibit high global trends for quality of governance. In contrast, domains with high challenges to adaptation ( 3 and 4) exhibit low global trends for quality of governance. Challenges domain 2 had medium outcomes for all socioeconomic elements most of the time, although mixed outcomes appeared for quality of governance and global innovation capacity. Finally, the Low challenges domain 1 is the only one characterized by high global trends in agricultural productivity, governance, income per capita, educational attainment, and low global trends for extreme poverty.

Unexpected patterns emerged as well. In the CIB analysis of judgments from Panel A, innovation capacity turned out to be most related to challenges to mitigation, whereas it was identified as relevant to challenges to adaptation by us (in section 2) and other scholarly literature (see discussion in Online Resource 1). CIB analysis results indicated that high global trends for innovation capacity were consistent most clearly with decreasing challenges to mitigation (see Fig. 3, domain 4; however, Panel B arrived at a different finding as discussed in Online Resource 5). Moreover, the high trend for innovation capacity in domain 4 does not give rise to high trends for global income per capita, low global trends for extreme poverty, or high trends in global educational attainment. Such trends are all medium for domain 4, which could mean that income growth, reductions in extreme poverty, and improvements in educational attainment are uneven globally. These relationships are apparent from investigating the judgments in Panel A as described in section 4 of Online Resource 1. However, because this analysis for challenges to adaptation was conceptualized at a globally aggregated scale, differences in trends across large world regions cannot be distinguished explicitly.

Additionally, domains with low challenges to mitigation but differing challenges for adaptation (domain 1 versus 4 ) can be distinguished by quality of governance. While the challenges domain 1 has low global trends for energy and carbon intensity coupled with a high global trend for quality of governance, domain 4 decouples this outcome. Governance may be a key socioeconomic element distinguishing the high adaptation challenges of domain 4 from the low adaptation challenges of domain 1. This interpretation is echoed by the low trend for quality of governance among combinations binned in challenges domain 3. Panel B also reproduced these findings.

Finally, it may appear curious that among the 1,000 consistent combinations, the vast majority had medium trends for most elements relevant to challenges to adaptation. This 
finding may not necessarily be considered meaningful, as globally averaged outcomes can mask divergences in local outcomes that this analysis does not capture. Additionally, results can differ under different weighting assumptions and under different panel judgments. More discussion of these issues is in Online Resource 5.

Nevertheless, the above findings can be used to revisit the specific cross-impact judgments and written statements provided by experts in their elicitation questionnaires. This requires investigating the distinctive trends summarized in Fig. 3 with the CIB matrix to begin archetypal descriptions of each domain. This procedure is described in section 4 of Online Resource 1. Here we present sketches of possible SSPs for each domain, based on the general characteristics summarized in Fig. 3.

SSP1 This world has low challenges to mitigation, as global population growth, energy intensity, and carbon intensity are low. These outcomes are made possible by a global trend in high quality of governance, as good governance encourages conditions for high educational attainment and high agricultural productivity across countries. In turn, these educational and agricultural outcomes reinforce sustained decreases in extreme poverty and increases in income per capita. High educational attainment also decreases fertility. Such beneficial social outcomes result in low challenges to adaptation. Additionally, the combined effects of increased wealth and good governance hasten trends for decreased energy intensity, which in turn hastens low carbon intensity.

$\operatorname{SSP} 2 a^{7}$ This is a world where historical trends for socioeconomic elements continue by and large (i.e., the medium trend for the vast majority of elements). However, agricultural productivity gains do not keep pace with historical trends. Inertias that prevent greater agricultural productivity (in order of importance): continued water scarcity, quality of governance, rate of educational attainment, innovation capacity, and rate of energy-related technological change.

SSP3 This world has high challenges to mitigation, as global population growth, energy intensity, and carbon intensity are high. Challenges to adaptation are also high due to low agricultural productivity and low innovation capacity. These outcomes are due to a low global trend for quality of governance, as poor governance has negative effects permeating multiple socioeconomic elements. Weak states are less able to improve or maintain agricultural markets, regulate energy efficiency, or protect intellectual property. In turn, low innovation capacity keeps energy and carbon intensity high and agricultural productivity low.

SSP4 This world has low challenges to mitigation, where innovation capacity is high, energy-related technological change is fast, energy and carbon intensity are low, and population growth has a medium or low trend. However, in contrast to SSP1, the global quality of governance trend is low. Like SSP3, poor governance depresses the global trend for agricultural productivity, which increases challenges to adaptation. Additionally, low governance quality prevents substantial gains in educational attainment or income growth and prevents substantial decreases in extreme poverty. These qualities depict a divided world where innovation capacity and technological change are directed successfully toward mitigation challenges rather than toward the improvement of quality of life.

\footnotetext{
${ }^{7}$ For some elements in domain 2, there were mixed trends with no single trend being dominant. Thus an alternative SSP2b could also describe a world with mixed outcomes. Due to space limitations, see Online Resource 1 for an example narrative of SSP2b.
} 
SSP5 This world is similar to SSP2 in that the historical trends of many socioeconomic elements continue. However, when innovation capacity is low, this reinforces high trends for energy and carbon intensity and low trends for agricultural productivity. ${ }^{8}$

\subsection{Sensitivity of findings}

As suggested throughout the paper, there are multiple aspects of the study that potentially affect results. These include the expert judgments collected, the construction of metrics for binning combinations in the challenges space, and the decision of where to draw domain boundaries. Online Resource 5 examines the implications of alternative decisions for these. Nonetheless, under the equal-weight metric and domain boundaries featured in this paper, results based on judgments from Panel B corroborated many of the findings for Panel A.

\section{Conclusion}

This study developed and demonstrated a systematic and traceable approach for developing new socioeconomic pathways at the globally aggregated scale. Its findings are suggestive for future research on SSPs, and its analytical approach is instructive for scenario development in general. This study involved a survey of 25 experts and 19 expert elicitations, as well as the development of metrics for binning combinations of socioeconomic trends in the challenges space. In completing this study, we addressed four key questions for fleshing out SSPs. First, using an expert survey, we identified 13 elements that should be prioritized for defining challenges to mitigation and adaptation at a globally aggregated scale.

Second, we demonstrated how different outcomes across these elements could be combined to derive new socioeconomic pathways that are internally consistent. When three categories of broad outcomes (low, medium, or high long-term trends) are assigned to each of the 13 elements, there are over 1.5 million ways to combine outcomes. The sheer number of possible ways to combine potential trends is a cautionary sign that selective investigation of the vast space of possibilities through IAM alone may be insufficient for identifying all policy relevant futures. For versions of socioeconomic pathways that are even richer than the ones investigated here, the space of scenario possibilities increases exponentially. This should be a genuine concern as versions of the "basic" global SSPs are "extended" to include additional socioeconomic elements (see O'Neill et al. 2013). Thus, analytical tools in addition to IAM (e.g., CIB) should be used to search for policy relevant futures and to augment assessments of internal consistency.

Third, we developed metrics for quantifying challenges to mitigation and to adaptation represented by 1,000 combinations of socioeconomic trends with the best internal consistency. We then used the metrics to bin the 1,000 combinations into five domains of the challenges space and found multiple cases that fell into each domain. As shown in Online Resource 5, decisions for what elements to assign to the challenge axes, as well as how to weight the elements for a composite index, can strongly affect scenario classification across domains.

Fourth, we conducted a meta-analysis on combinations in each domain to find archetypal and unexpected trends. A number of archetypal findings were confirmed by the judgments

\footnotetext{
${ }^{8}$ Although the population and governance trends for domain 5 are also distinctive compared to other domains, in this CIB analysis and under the conditions for binning, these distinctions simply reflect that combinations binned in domain 5 describe worlds that could not be classified in domains 2 or 3 . In other words, there is no particular logic that justifies the high trends for population or quality of governance.
} 
provided by both expert panels $\mathrm{A}$ and $\mathrm{B}$ : challenges to mitigation and to adaptation typically (although not exclusively) co-varied; domains with high challenges to mitigation (3 and 5) had high trends for population, energy intensity, and carbon intensity and had low trends for agricultural productivity; domain 2 generally exhibited a continuation of historical trends (i.e., a prevalence of medium trends).

Unexpected findings include the importance of quality of governance for adaptation challenges. A low outcome for quality of governance has compound direct and indirect negative effects on extreme poverty and educational attainment that distinguish domains with high adaptation challenges ( 3 and 4 ) versus low adaptation challenges ( 1 and 5). This finding was reproduced across judgments provided by both expert Panels A and B. In addition, we found unexpected results for domain 4 related to innovation capacity. Although literature around the development of the SSPs suggests that high innovation capacity may be just as important (if not more so) for addressing adaptation challenges, under the judgments assembled for Panel A, domain 4 can describe worlds where innovation capacity is applied successfully only in a one-sided manner toward mitigation challenges. With the findings of this meta-analysis, we then revisited the collection of expert judgments to delineate five possible starting points for narratives for the SSPs.

The findings of this study are suggestive for future research on SSPs, and they highlight three major questions. First, what metrics should be used to classify scenarios as depicting high versus low challenges to mitigation and adaptation? In our view, metrics for this purpose should be composite and pertain to multiple socioeconomic characteristics. However, how should such characteristics be combined? The main paper featured results assuming the simplest case of an additive, equal-weighted metric. Online Resource 5 shows that a differentially weighted metric could also be applied. Under differential weighting, there can be even more cases of mixed challenge worlds (domains 4 and 5) to investigate.

Second, could additional insights for challenges to mitigation and adaptation be gained by conducting a similar analysis at a regionally disaggregated scale, rather than a globally averaged scale? The prevalence of medium global trends for many elements relevant for challenges to adaptation may mask divergences occurring at more localized scales.

Third, the internally consistent logic of domain 4 (according to the judgments of Panel A) raises a host of questions. In this analysis, innovation capacity and quality of governance were treated qualitatively due to the many dimensions of these elements and the restriction to conceptualize their meaning and influences at the globally aggregated scale. Our findings suggest it would be worthwhile for future research to disaggregate specific dimensions of innovation capacity and quality of governance. Details for these elements may matter differentially for challenges to adaptation or mitigation.

Finally, our analytical approach is instructive for scenario development in general, as it made a number of assumptions that are part of that process more obvious. Assumptions for any scenario exercise include decisions for how many experts to consult, which experts to consult, how to collect their judgments, how to verify the internal consistency of their judgments, and how to handle differences in expert opinion. Under the new SSP framework, additional decisions must be made for how to classify particular scenarios as representative of a particular domain within the challenges space (i.e., of a particular SSP archetype), and how to assess scenarios developed at different geographic scales. Decisions at each of these steps can potentially influence the conclusions of a scenario study, which is why it is important for such decisions to be traceable, or publicly accessible. In our view, the scientific quality and political legitimacy of scenario analyses will be improved by integrating systematic and traceable methods (e.g. CIB). Lloyd and Schweizer (2013) discuss these issues at length and how publicly accessible methods can make scenario analyses more responsive to improvements in data and theory. On this note, our process could have been improved further with 
a less structured survey for the prioritization of scenario elements, the participation of more experts (both in the survey and in the elicitation), through more iterations with experts, and potentially by investigating more than 1,000 internally consistent scenarios. These limitations notwithstanding, we demonstrated how new scenarios could be developed with systematic and traceable methods. We also determined findings that are informative and suggestive for continued research on the SSPs.

Acknowledgments The National Center for Atmospheric Research (NCAR) is funded by the National Science Foundation and managed by the University Corporation for Atmospheric Research. Vanessa Schweizer would like to thank the Integrated Science Program at NCAR for supporting her travel to scientific meetings regarding SSPs as well as for conducting a face-to-face elicitation workshop. The authors would also like to thank the organizers of the Fourth Annual Meeting of the Integrated Assessment Modeling Consortium for facilitating research activities, and Wolfgang Weimer-Jehle and Hannah Kosow for helpful discussions.

Open Access This article is distributed under the terms of the Creative Commons Attribution License which permits any use, distribution, and reproduction in any medium, provided the original author(s) and the source are credited.

\section{References}

Alcamo J (2008) Introduction: the case for scenarios of the environment. In: Alcamo J (ed) Environmental futures: the practice of environmental scenario analysis. Elsevier, San Francisco, pp 1-11

Alcamo J, Henrichs T (2008) Towards guidelines for environmental scenario analysis. In: Alcamo J (ed) Environmental futures: the practice of environmental scenario analysis. Elsevier, San Francisco, pp 13-35

Ebi KL, Hallegatte S, Kram T et al (2013) A new scenario framework for climate change research: background, process, and future directions. Clim Chang. doi:10.1007/s10584-013-0912-3

Girod B, Flueeler T (2009) Future IPCC scenarios - lessons learned and challenges to scenario building in climate change policy. Paper presented at the International Energy Workshop, Venice

IAMC (Integrated Assessment Modeling Consortium) (2012) SMA-SSP scenarios workshop. http:// www.iamconsortium.org/ (accessed 14 January 2013)

Kriegler E, Edmonds J, Hallegatte S, Ebi K, Kram T, Riahi K, Winkler H, van Vuuren DP. (submitted for this issue). A new scenario framework for Climate Change Research: The concept of Shared Policy Assumptions. Climatic Change

Lloyd EA, Schweizer VJ (2013) Objectivity and a comparison of methodological scenario approaches for climate change research. Accepted for publication in Synthese. doi:10.1007/s11229-013-0353-6

Moss RH, Edmonds JA, Hibbard KA et al (2010) The next generation of scenarios for climate change research and assessment. Nature 463:747-756

Nakicenovic N, Alcamo J, Davis G et al (2000) Special report on emissions scenarios. Cambridge University Press, New York

O’Neill BC, Carter T, Ebi KL et al (2012) Meeting report of the workshop on the nature and use of new socioeconomic pathways for climate change research. University Corporation for Atmospheric Research. http://www.isp.ucar.edu/socio-economic-pathways. Accessed 22 May 2012

O’Neill BC, Kriegler E, Riahi K et al (2013) A new scenario framework for Climate Change Research: The concept of Shared Socioeconomic Pathways. Clim Chang. doi:10.1007/s10584-013-0905-2

Rounsevell MDA, Metzger MJ (2010) Developing qualitative scenario storylines for environmental change assessment. WIREs Clim Chang 1:606-619

Schweizer VJ, Kriegler E (2012) Improving environmental change research with systematic techniques for qualitative scenarios. Environ Res Lett 7:044011

Smit B, Wandel J (2006) Adaptation, adaptive capacity and vulnerability. Glob Environ Chang 16:282-292

van Vuuren DP, Edmonds J, Kainuma M et al (2011) The representative concentration pathways: an overview. Clim Chang 109:5-31

van Vuuren DP, Kriegler E, O'Neill BC et al (2013) A new scenario framework for Climate Change Research: scenario matrix architecture. Clim Chang. doi:10.1007/s10584-013-0906-1

von Reibnitz U (1988) Scenario techniques. McGraw-Hill Book Company GmbH, St. Louis

Weimer-Jehle W (2006) Cross-impact balances: a system-theoretical approach to cross-impact analysis. Technol Forecast Soc Chang 73:334-361 